\title{
Expected performance of the ATLAS Inner Tracker upgrade
}

\author{
Simon Viel ${ }^{* \dagger}$ \\ on behalf of the ATLAS Collaboration \\ Lawrence Berkeley National Lab. (US) \\ E-mail: sviel@cern.ch
}

\begin{abstract}
The design of the ATLAS Inner Tracker upgrade is underway. This tracking detector, consisting of silicon pixel and strip modules, will replace the current ATLAS Inner Detector to reconstruct tracks from charged particles produced at the very high collision rate expected from the High-Luminosity Large Hadron Collider. The latest Inner Tracker designs considered, and recent expected performance results from simulation are presented.
\end{abstract}

38th International Conference on High Energy Physics

3-10 August 2016

Chicago, USA

\footnotetext{
* Speaker.

${ }^{\dagger}$ The author acknowledges support from the U.S. Department of Energy and the Natural Sciences and Engineering Research Council of Canada.
} 


\section{Introduction}

In 2024-2026, the Large Hadron Collider (LHC) will be upgraded to become the HighLuminosity LHC (HL-LHC), foreseen to deliver an average of up to 200 proton-proton collisions every 25 nanoseconds. With an expected dataset of up to $3000 \mathrm{fb}^{-1}$ at $\sqrt{s}=14 \mathrm{TeV}$ accumulated over 10 years of HL-LHC operation, the discovery reach of the ATLAS experiment [1] for physics beyond the Standard Model will be significantly improved beyond what is possible with the LHC dataset [2]. This is particularly true for new processes with small production cross-sections, for example due to small couplings to Standard Model particles. In the event of a discovery, measuring the properties of new particles in this large dataset would give physicists the ability to distinguish between different theoretical models attempting to explain the new phenomena.

The HL-LHC dataset will also allow particle physicists to deepen our understanding of Standard Model processes. Differential cross-section measurements such as Higgs boson production and phenomena like same-sign WW production will be performed, looking for deviations with respect to theoretical predictions. Precision measurements of Higgs boson couplings will also become possible [3], including rare decays such as $H \rightarrow Z \gamma, H \rightarrow \mu^{+} \mu^{-}$, and perhaps even $H \rightarrow c \bar{c}$. Measurements will be carried out in all major Higgs boson production modes: gluon fusion, vector boson fusion (VBF), $V H, t \bar{t} H, t H$ and $b \bar{b} H$. The search for di-Higgs production will take centre stage, mainly in an attempt to measure the triple-Higgs self-coupling but also in searches for di-Higgs production mechanisms beyond the Standard Model.

Many upgrades to the ATLAS detector will be necessary in order to operate in the very highrate environment of the HL-LHC. These scheduled upgrades include a complete replacement of the ATLAS Inner Detector (ID), with a new Inner Tracker (ITk). The innermost region of ATLAS will be instrumented with the latest generation of high-rate, low-mass, radiation-hard silicon detectors. Excellent tracking acceptance and resolution, collision vertex identification, and the rejection of hadronic jets coming from simultaneous collisions other than the one of interest (pileup) are essential features of the ITk. Given the scale of this project, construction must begin to ramp up in mid-2018, and the final steps of technical design and prototyping are underway.

\section{Inner Tracker layout: latest design options}

Two main Inner Tracker layout design options, shown in Figure 1, are currently under review. Both designs have the same silicon strip detector, with four stereo barrel layers, in addition to six stereo endcap disks on each side of the detector providing coverage up to pseudorapidity $|\eta|=2.7$. The silicon pixel detector also has the same number of layers in the two layouts: five layers in the barrel, and four concentric ring assemblies, called ring layers, in each endcap. However, the orientation of the pixel modules in the forward region of the barrel is different. Endcap ring positions have been optimized in each layer to independently provide a required number of measurements for all tracks originating from the collision region, i.e. within $15 \mathrm{~cm}$ of the detector origin.

One of the detector designs features extended pixel barrel layers, to achieve tracking coverage up to $|\eta|=4$ using long staves. In the forward region of the detector, tracks cross the sensors at small incidence angle, resulting in the measurement of long pixel clusters [4]. This is expected to bring improvements in pattern recognition for track reconstruction and pileup jet rejection at 

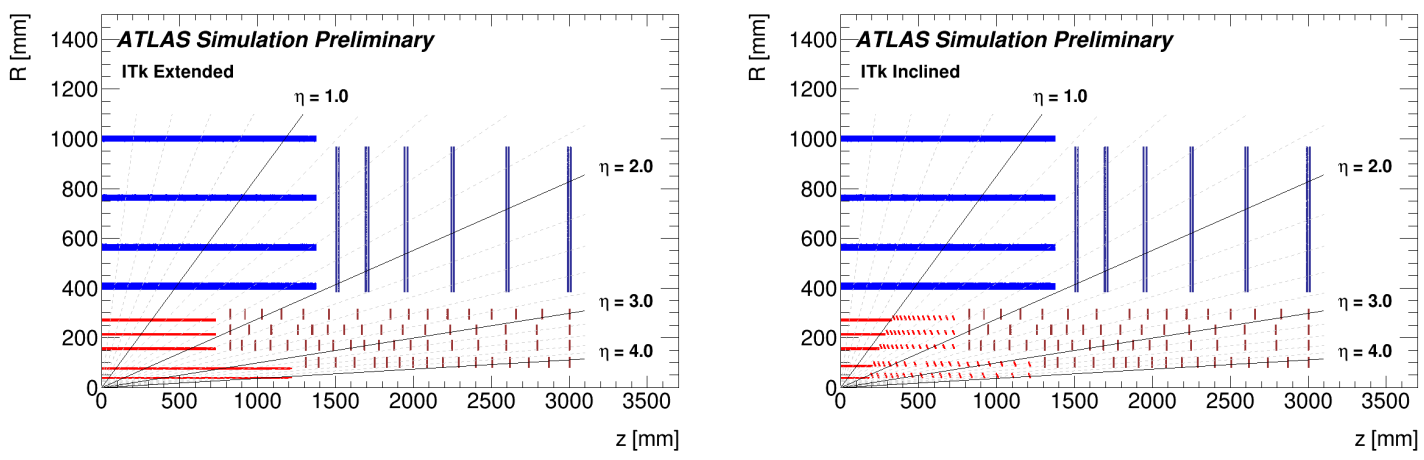

Figure 1: The main ITk layout designs under consideration, with extended pixel barrel layers (left) and inclined pixel barrel layers (right). The differences between the two designs are in the forward region of the pixel detector (red). The stereo strip detector (blue) is the same in both options. [5]

very high pileup, as well as new algorithms for measuring the luminosity delivered to ATLAS by counting long clusters, and for particle identification using long cluster shapes and $d E / d X$.

In contrast, the ITk layout with inclined pixel barrel layers makes use of an innovative mechanical design allowing to place pixel modules in the forward barrel at an angle with respect to the beam pipe. Compared with the alternative, this layout measures a higher number of spacepoints on each track, with tracks from the luminous region crossing the sensors at approximately normal incidence. Hermetic tracking coverage up to $|\eta|=4$ is achieved with a smaller silicon area and fewer pixel chips than with extended barrel layers, although since inclined modules are in general smaller, the total number of modules is slightly higher. This corresponds to a small reduction in material in the most forward region of the detector. The ITk community is actively evaluating the merits of the two proposed layouts, mainly based on the expected performance of the detector. A decision between the two options for the ITk pixel detector is expected in early 2017.

\section{Selected expected performance results}

Increasing the tracking coverage to the forward region of the detector significantly improves the detector capabilities [2] for vertex identification, pileup jet rejection, electron and photon identification, muon acceptance, missing transverse momentum $\left(E_{\mathrm{T}}^{\mathrm{miss}}\right)$ resolution and $b$-tagging performance. Many of these studies are based on three older Inner Tracker layout designs presented in [2]: the Reference, Middle and Low scenarios covering up to $|\eta|=4.0,3.2$ and 2.7 respectively ${ }^{1}$. The Reference scenario also includes improvements to the forward calorimeters of ATLAS.

Selected expected performance results are shown in Figure 2. For the benchmark processes $Z \rightarrow \mu^{+} \mu^{-}$and VBF $H \rightarrow \gamma \gamma$, the vertex identification efficiency improves by about $10 \%$ when forward tracks are measured, and is robust against disabling $10 \%$ of ITk silicon modules. Pileup jets are rejected using tracking confirmation (TC) algorithms, based on the momentum of tracks within a jet which are associated with the primary vertex. Measuring tracks at high $|\eta|$ improves the range of this technique. Pileup jet rejection also allows to improve the $E_{\mathrm{T}}^{\text {miss }}$ resolution, with the best improvements being observed for the most energetic collision events.

\footnotetext{
${ }^{1}$ Updated tracking performance results based on the latest ITk layout designs are available in [5]
} 

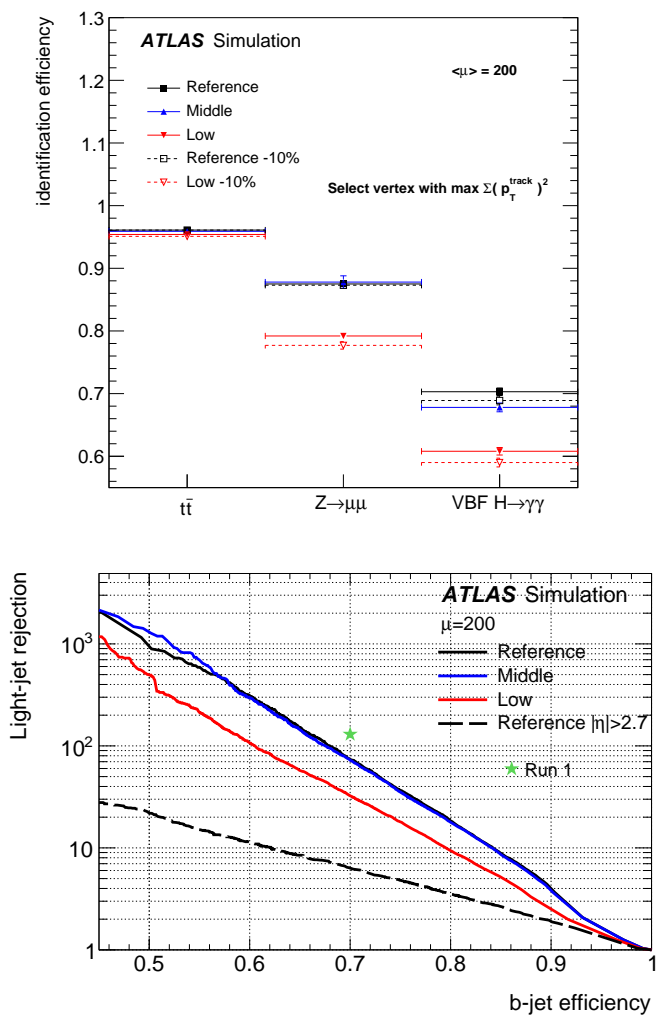

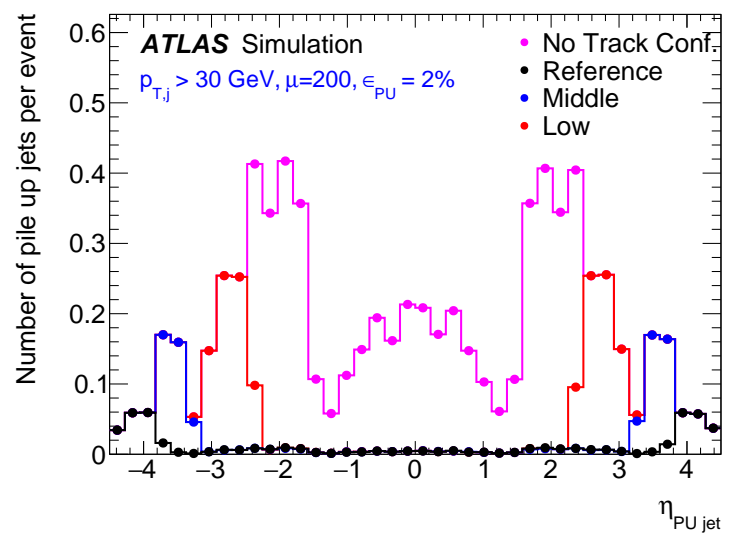

Figure 2: Top left: Vertex identification efficiency for $t \bar{t}, Z \rightarrow \mu^{+} \mu^{-}$and VBF $H \rightarrow \gamma \gamma$. Top right: Number of pileup jets per event without TC, and applying TC algorithms tuned to give $2 \%$ pileup jet acceptance. Bottom left: Light-flavour jet rejection versus $b$-jet efficiency for the MV1 tagging algorithm. [2]

Figure 2 also shows the $b$-tagging performance of the ITk, evaluated using ATLAS Run-1 algorithms. For $|\eta|<2.7$, the ITk performance in the Reference and Middle layouts is close to the Run-1 operating point, in spite of much higher pileup at the HL-LHC. Improving the tracking coverage up to $|\eta|=4$ makes forward $b$-tagging possible. These results will be improved by the use of new algorithms optimized for high pileup.

\section{Conclusion}

Two design concepts for the ATLAS ITk upgrade towards the HL-LHC are considered, with different module orientation choices in the forward pixel barrel. Both options offer hermetic tracking coverage up to $|\eta|=4$, motivated by the improved vertex identification, pileup jet rejection, as well as the identification, acceptance and resolution capabilities for physics objects. Forward tracking maximizes the expected performance of the upgraded ATLAS detector at the HL-LHC.

\section{References}

[1] ATLAS Collaboration, JINST 3 (2008) S08003

[2] ATLAS Collaboration, CERN-LHCC-2015-020 (2015)

[3] ATLAS Collaboration, ATL-PHYS-PUB-2014-016 (2014)

[4] S. Viel et al., Nucl. Instrum. Methods Phys. Res. A 831 (2016) 254-259

[5] ATLAS Collaboration, ATL-PHYS-PUB-2016-025 (2016) 